\title{
Antidiabetic medication-induced acute interstitial nephritis: case report and literature search
}

\author{
NADIA CHAUDHURY, ${ }^{1}$ ALEXANDROS L LIARAKOS, ${ }^{1}$ KISHORE GOPALAKRISHNAN, ${ }^{2}$ WAQAR AYUB, ${ }^{3}$ \\ NARASIMHA MURTHY, ${ }^{1}$ RANGANATHA RAO ${ }^{1}$
}

Key words: GLP-1 receptor agonists, liraglutide, renal impairment, acute kidney injury, nephrology, renal, diabetes

\section{Introduction}

Liraglutide, a glucagon-like peptide-1 (GLP-1) receptor agonist, is a recognised treatment for type 2 diabetes mellitus (T2DM). It mimics human GLP-1 and works by augmenting insulin secretion, inhibiting glucagon secretion and inhibiting gastric acid secretion. ${ }^{1}$ It has been shown to not only improve glycaemic control in people with diabetes, but also result in weight loss, reduced hypoglycaemic episodes, reduced albuminuria, reduced progression to macroalbuminuria and reduced incidence of myocardial infarction and stroke events. ${ }^{2-5}$ Gastrointestinal upset is the commonest reported side effect, which occurs in up to $56 \%$ of patients in clinical trials. Furthermore, BNF recommends avoiding liraglutide treatment in end-stage renal disease/estimated glomerular filtration rate (eGFR) $<15 \mathrm{~mL} / \mathrm{min} / 1.73 \mathrm{~m}^{2}$ (depending on brand), due to the increased risk of adverse events.

We present a rare case of a female with chronic kidney disease (CKD), whose treatment with liraglutide was associated with rapid deterioration of renal function and tubulointerstitial nephritis. Our literature search highlighted one previous case, thus we would like to raise awareness of this potential rare side effect of liraglutide treatment. ${ }^{6}$ We have further conducted a literature search of all case reports noting associations of glucose-lowering therapies with acute interstitial nephritis to raise awareness of this potential complication.

\section{Case history}

A 59-year-old woman with T2DM and CKD stage 3 (G3b A1),

WISDEM Centre, Department of Endocrinology and Diabetes, University Hospitals Coventry and Warwickshire NHS Trust

2 Department of Histopathology, University Hospitals Coventry and Warwickshire NHS Trust

3 Department of Renal Medicine, University Hospitals Coventry and Warwickshire NHS Trust

Address for correspondence: Dr Nadia Chaudhury University Hospitals Coventry and Warwickshire NHS Trust, Clifford Bridge Road, Coventry, CV2 2DX, UK

E-mail: nadia-chaudhury@hotmail.co.uk

https://doi.org/10.15277/bjd.2021.321

Br J Diabetes 2021;21:228-232
Table 1 Renal function and glycaemic control pre- and post-liraglutide treatment

\begin{tabular}{lll}
\hline & Liraglutide started & Liraglutide stopped \\
Creatinine & 136 & 248 \\
eGFR & 35 & 17 \\
$\mathrm{HbA}_{1 \mathrm{c}}$ & 58 & 55
\end{tabular}

*eGFR, estimated glomerular filtration rate; $\mathrm{HbA}_{1 c}$, haemoglobin $\mathrm{A}_{1 \mathrm{c}}$.

maintained stable glycaemic control with $\mathrm{HbA}_{1 \mathrm{c}} 58$ on linagliptin $5 \mathrm{mg}$ once daily and reduced carbohydrate diet. Medication history also included amlodipine $5 \mathrm{mg}$ and atorvastatin $20 \mathrm{mg}$. She was started on liraglutide in January 2019 to improve her metabolic control further. Linagliptin treatment was discontinued, given that the combination of linagliptin and liraglutide is unlikely to provide synergistic effects and is not cost effective. Despite improvement in glycaemia, rapid deterioration in renal function was noted subsequent to starting liraglutide (Table 1 and Figure 1).

There was no other explanation for the drop in eGFR: she did not experience diarrhoea, nausea or vomiting. Weight and BMI had remained stable throughout liraglutide treatment at $70 \mathrm{~kg}$ and 24.3 $\mathrm{kg} / \mathrm{m}^{2}$, respectively. Furthermore, blood pressure remained stable at around 120/70 $\mathrm{mm} \mathrm{Hg}$. She had remained euvolaemic whilst on liraglutide treatment. She had not taken NSAIDs. Urinalysis was bland. Complement C3 and C4, double-stranded DNA 1 (dsDNA1), anti-neutrophil cytoplasm antibodies (ANCA), myeloperoxidase (MPO) and anti-proteinase 3 antibody (PR3) were all negative. Ultrasound of the kidney showed normal kidneys bilaterally (right $10.7 \mathrm{~cm}$, left $10.4 \mathrm{~cm}$ ) and no evidence of obstruction. Moreover, there was no family history of renal disease.

As the rapid drop in eGFR was only noted after commencing liraglutide, it was postulated that the loss in renal function may be due to liraglutide initiation. Unfortunately, due to a delay in the medical appointments, the only available eGFR results were prior to and at 5 months after start of treatment, thus complicating matters further. The eGFR was $35 \mathrm{~mL} / \mathrm{min} / 1.73 \mathrm{~m}^{2}$ prior to starting liraglutide and dropped rapidly to $17 \mathrm{~mL} / \mathrm{min} / 1.73 \mathrm{~m}^{2}$ when measured five months later - a significant fall of $51 \%$. It was therefore decided to stop liraglutide treatment in May 2019 and her linagliptin was restarted.

Renal biopsy was performed in July 2019 (Figure 2). Significant interstitial fibrosis and tubular atrophy (IFTA) were present, with 
Figure 1. Change in estimated glomerular filtration rate (eGFR) with time. Our case showed rapid deterioration in renal function on initiation of liraglutide treatment. This unfortunately did not improve once liraglutide was stopped.

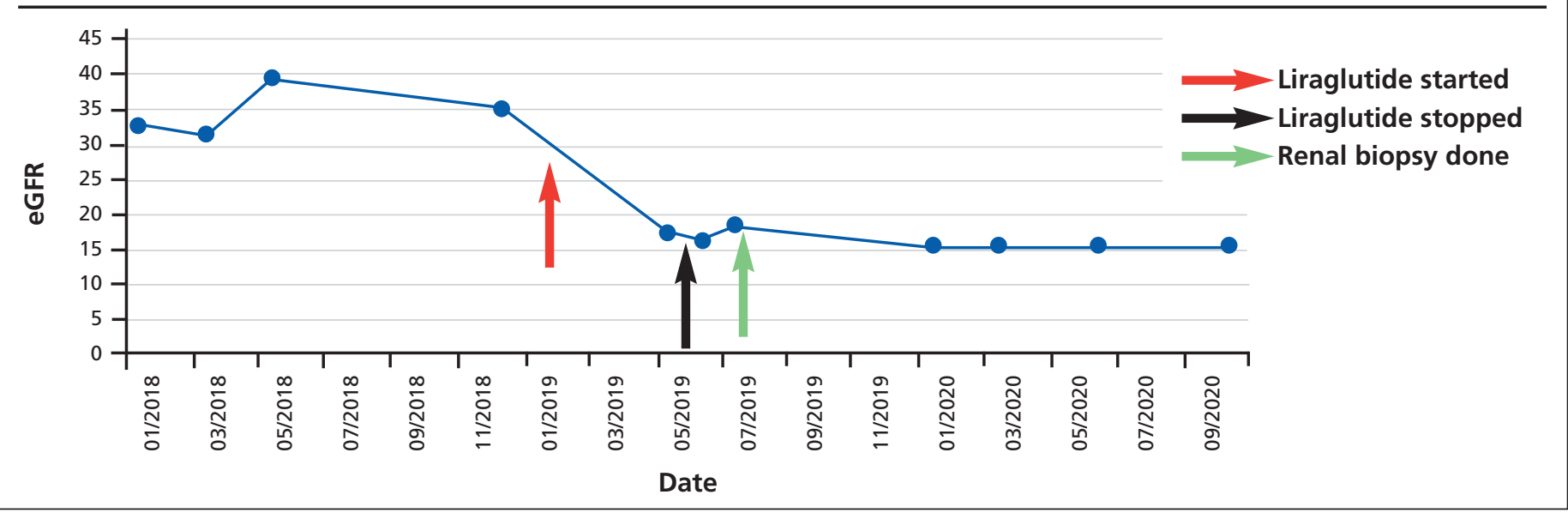

Figure 2. Renal biopsy showing marked interstitial fibrosis and tubular atrophy involving two-thirds of the cortical tissue. There is prominent interstitial infiltrate, including substantial numbers of eosinophils suggestive of tubulointerstitial nephritis. Mild focal tubulitis is evident.

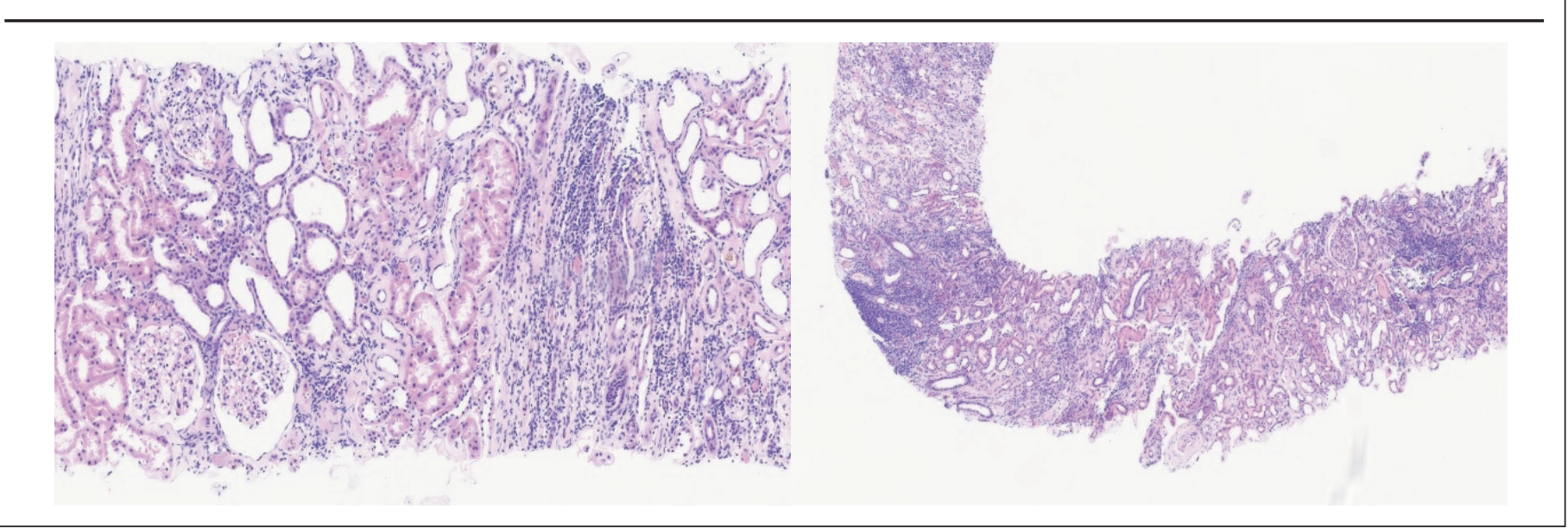

profound interstitial inflammatory infiltrate and mild focal tubulitis. All these findings heavily supported the diagnosis of acute interstitial nephritis. Furthermore, mild glomerular mesangial expansion and arteriolar hyalinosis was seen, suggestive but not specific for a diagnosis of diabetic nephropathy. We therefore concluded that the kidney biopsy showed significant acute interstitial nephritis with mild diabetic nephropathy.

Despite stopping liraglutide, her renal function did not improve (Figure 1). She progressed to end stage renal disease. Steroid treatment was considered but not deemed beneficial due to severe IFTA and her diabetes. She has started peritoneal dialysis and is awaiting renal transplant.

\section{Discussion}

Acute interstitial nephritis is a common cause of acute kidney injury $(\mathrm{AKI})$, and has been identified in the diagnosis of $12.9 \%$ of kidney biopsies from patients with $\mathrm{AKI} .^{7}$ The main cause of acute interstitial nephritis in the developed world is medications, which contribute to $>70 \%$ of cases. ${ }^{8}$ Antibiotics, NSAIDs, anti-inflammatory agents, anticonvulsants, diuretics and proton pump inhibitors are the most common culprits.9,10 Other causes of interstitial nephritis include infections, autoimmune disorders, systemic diseases, metabolic causes and environmental exposure. ${ }^{11}$ The presentation of druginduced acute interstitial nephritis is highly variable. ${ }^{12}$ The classic triad of symptoms of rash, fever and eosinophilia are only witnessed in $<10 \%$ of patients. ${ }^{9}$ Laboratory tests and imaging are usually unhelpful in the diagnosis of drug-induced acute interstitial nephritis as they lack both sensitivity and specificity. ${ }^{9,12}$ Renal biopsy is the gold standard investigation to make a definitive diagnosis. Main histological findings include interstitial inflammation, comprising primarily of lymphocytes and monocytes, and tubulitis is evident. If the nephritis continues to deteriorate, chronic appearances of interstitial fibrosis and tubular atrophy may be present. ${ }^{9}$

Our case highlights a person with stable diabetes and established CKD whose kidney function rapidly deteriorated further after liraglutide initiation. People with diabetes and CKD3 are expected 
Table 2 Cases of antidiabetic medication-induced acute interstitial nephritis to date

\begin{tabular}{|c|c|c|c|c|c|c|c|c|c|}
\hline Class of Drug & Drug name & Author & Patient & $\begin{array}{l}\text { Initial renal } \\
\text { function }\end{array}$ & $\begin{array}{l}\text { Time to } \\
\text { presentation } \\
\text { at hospital }\end{array}$ & $\begin{array}{l}\text { Renal function } \\
\text { post GLP-1 } \\
\text { introduction }\end{array}$ & Renal & $\begin{array}{l}\text { Treatment } \\
\text { biopsy }\end{array}$ & Recovery \\
\hline \multirow[t]{6}{*}{ GLP 1 RA } & Liraglutide & $\begin{array}{l}\text { Gariani et al } \\
2014^{1}\end{array}$ & $83 \mathrm{M}$ & $\begin{array}{l}\text { Creatinine } 2.14 \mathrm{mg} / \mathrm{dL} \\
\text { eGFR } 32 \mathrm{~mL} / \mathrm{min} / 1.73 \mathrm{~m}^{2}\end{array}$ & - & $\begin{array}{l}\text { Creatinine } 9.3 \mathrm{mg} / \mathrm{dL} \\
\text { eGFR } 6 \mathrm{~mL} / \mathrm{min} / 1.73 \mathrm{~m}^{2}\end{array}$ & Confirmed & $\begin{array}{l}\text { Stopped liraglutide. } \\
\text { Steroids and } \\
\text { transient dialysis }\end{array}$ & Partial recovery \\
\hline & Exenatide & $\begin{array}{l}\text { Dubois - } \\
\text { Laforgue et al } \\
2014^{2}\end{array}$ & $75 \mathrm{M}$ & Creatinine $130 \mu \mathrm{mol} / \mathrm{L}$ & 5 days & Creatinine $1148 \mu \mathrm{mol} / \mathrm{L}$ & Not done & $\begin{array}{l}\text { Stopped exenatide. } \\
\text { Haemodialysis for } \\
\text { 48h insulin therapy }\end{array}$ & $\begin{array}{l}\text { Full recovery } \\
-9 \text { days }\end{array}$ \\
\hline & Exenatide & $\begin{array}{l}\text { Bhatti et al } \\
2010^{3}\end{array}$ & $65 F$ & $\begin{array}{l}\text { Creatinine } 77 \mu \mathrm{mol} / \mathrm{L} \\
\text { eGFR } 66 \mathrm{~mL} / \mathrm{min} / \mathrm{m}^{2}\end{array}$ & 9 weeks & $\begin{array}{l}\text { Creatinine } 393 \mu \mathrm{mol} / \mathrm{L} \\
\text { eGFR } 10 \mathrm{~mL} / \mathrm{min} / \mathrm{m}^{2}\end{array}$ & Not done & $\begin{array}{l}\text { Liraglutide stopped. } \\
\text { Prednisolone }\end{array}$ & $\begin{array}{l}\text { Partial recovery } \\
-6 \text { weeks }\end{array}$ \\
\hline & Exenatide & $\begin{array}{l}\text { Nandokaban } \\
\text { et al } 2013^{4}\end{array}$ & $58 \mathrm{M}$ & $\begin{array}{l}\text { Creatinine } 120 \mu \mathrm{mol} / \mathrm{L} \\
\text { eGFR } 59 \mathrm{~mL} / \mathrm{min} / 1.73 \mathrm{~m}^{2}\end{array}$ & 2 months & $\begin{array}{l}\text { Serum creatinine } \\
209 \mu \mathrm{mol} / \mathrm{L} \\
\text { eGFR } 39 \mathrm{~mL} / \mathrm{min} / 1.73 \mathrm{~m}^{2}\end{array}$ & Confirmed & $\begin{array}{l}\text { Stopped exenatide. } \\
\text { Prednisolone }\end{array}$ & $\begin{array}{l}\text { Partial recovery } \\
-4 \text { months }\end{array}$ \\
\hline & Semaglutide & $\begin{array}{l}\text { Leehey et al } \\
2021^{5}\end{array}$ & $\sim 80 \mathrm{~F}$ & $\begin{array}{l}\text { Creatinine } 1.59 \mathrm{mg} / \mathrm{dL} \\
\text { eGFR } 30 \mathrm{~mL} / \mathrm{min} / 1.73 \mathrm{~m}^{2}\end{array}$ & 5 months & $\begin{array}{l}\text { Creatinine } 3.50 \mathrm{mg} / \mathrm{dL} \\
\text { eGFR } 11 \mathrm{~mL} / \mathrm{min} / 1.73 \mathrm{~m}^{2}\end{array}$ & Confirmed & $\begin{array}{l}\text { Discontinued } \\
\text { semaglutide }\end{array}$ & No recovery \\
\hline & Dulaglutide & $\begin{array}{l}\text { Taylor et al } \\
2018^{6}\end{array}$ & $63 \mathrm{~F}$ & $\begin{array}{l}\text { Creatinine } 1.6 \mathrm{mg} / \mathrm{dL} \\
\text { eGFR } 34 \mathrm{~mL} / \mathrm{min} / 1.73 \mathrm{~m}^{2}\end{array}$ & 1 month & $\begin{array}{l}\text { Creatinine } 3.4 \mathrm{mg} / \mathrm{dL} \\
\text { eGFR } 13.7 \mathrm{~mL} / \mathrm{min} / 1.73 \mathrm{~m}^{2}\end{array}$ & Not done & $\begin{array}{l}\text { Discontinued } \\
\text { dulaglutide }\end{array}$ & $\begin{array}{l}\text { Full recovery - } \\
4 \text { weeks }\end{array}$ \\
\hline \multirow[t]{3}{*}{$\begin{array}{l}\text { SGLT2 } \\
\text { inhibitors }\end{array}$} & Empagliflozin & $\begin{array}{l}\text { Ryan et al } \\
2021^{7}\end{array}$ & $63 \mathrm{~F}$ & Creatinine $60 \mu \mathrm{mol} / \mathrm{L}$ & 6 weeks & Creatinine $381 \mu \mathrm{mol} / \mathrm{L}$ & Confirmed & $\begin{array}{l}\text { Discontinued } \\
\text { empagliflozin. } \\
\text { Prednisolone }\end{array}$ & $\begin{array}{l}\text { Partial recovery } \\
-8 \text { weeks }\end{array}$ \\
\hline & Empagliflozin & $\begin{array}{l}\text { Bnaya et al } \\
2020^{8}\end{array}$ & $67 F$ & $\begin{array}{l}\text { Creatinine } 0.9 \mathrm{mg} / \mathrm{dL} \\
\text { eGFR } 66 \mathrm{~mL} / \mathrm{min} / 1.73 \mathrm{~m}^{2}\end{array}$ & 1 week & $\begin{array}{l}\text { Creatinine } 3.19 \mathrm{mg} / \mathrm{dL} \\
\text { eGFR } 15 \mathrm{~mL} / \mathrm{min} / 1.73 \mathrm{~m}^{2}\end{array}$ & Confirmed & $\begin{array}{l}\text { Haemodialysis, } \\
\text { prednisolone }\end{array}$ & $\begin{array}{l}\text { Partial recovery } \\
-3 \text { months. }\end{array}$ \\
\hline & Canagliflozin & $\begin{array}{l}\text { Gribben et al } \\
2021^{9}\end{array}$ & $51 \mathrm{M}$ & $\begin{array}{l}\text { Creatinine } 1.5 \mathrm{mg} / \mathrm{dL} \\
\text { eGFR } 63 \mathrm{~mL} / \mathrm{min} / 1.73 \mathrm{~m}^{2}\end{array}$ & 2 weeks & $\begin{array}{l}\text { Creatinine } 11.6 \mathrm{mg} / \mathrm{dL} \\
\text { eGFR } 6 \mathrm{~mL} / \mathrm{min} / 1.73 \mathrm{~m}^{2}\end{array}$ & $\begin{array}{l}\text { Not } \\
\text { diagnostic - } \\
\text { not enough } \\
\text { tissue } \\
\text { obtained }\end{array}$ & $\begin{array}{l}\text { Discontinued } \\
\text { canagliflozin. } \\
\text { IV fluids. } \\
\text { Haemodialysis }\end{array}$ & $\begin{array}{l}\text { Deterioration } \\
\text { of kidney } \\
\text { function }\end{array}$ \\
\hline \multirow[t]{3}{*}{$\begin{array}{l}\text { DPP4 } \\
\text { Inhibitors }\end{array}$} & Sitagliptin & $\begin{array}{l}\text { Lin et al } \\
2014^{10}\end{array}$ & $69 \mathrm{M}$ & $\begin{array}{l}\text { Creatinine } 1.07 \mathrm{mg} / \mathrm{dL} \\
\text { eGFR } 69 \mathrm{~mL} / \mathrm{min} / 1.73 \mathrm{~m}^{2}\end{array}$ & 4 weeks & $\begin{array}{l}\text { Creatinine } 4.95 \mathrm{mg} / \mathrm{dL} \\
\text { eGFR } 12 \mathrm{~mL} / \mathrm{min} / 1.73 \mathrm{~m}^{2}\end{array}$ & Confirmed & $\begin{array}{l}\text { Discontinued } \\
\text { sitagliptin. } \\
\text { Haemodialysis. } \\
\text { Prednisolone }\end{array}$ & $\begin{array}{l}\text { Partial recovery } \\
-3 \text { weeks }\end{array}$ \\
\hline & Sitagliptin & $\begin{array}{l}\text { Alsaad et al } \\
2016^{11}\end{array}$ & $56 \mathrm{M}$ & $\begin{array}{l}\text { Creatinine } 1.5 \mathrm{mg} / \mathrm{dL} \\
\text { eGFR } 51 \mathrm{~mL} / \mathrm{min} / 1.73 \mathrm{~m}^{2}\end{array}$ & 2 weeks & $\begin{array}{l}\text { Creatinine } 2.2 \mathrm{mg} / \mathrm{dL} \\
\text { eGFR } 33 \mathrm{~mL} / \mathrm{min} / 1.73 \mathrm{~m}^{2}\end{array}$ & Confirmed & $\begin{array}{l}\text { Discontinued } \\
\text { sitagliptin. } \\
\text { Prednisolone }\end{array}$ & $\begin{array}{l}\text { Full recovery - } \\
6 \text { weeks }\end{array}$ \\
\hline & Alogliptin & $\begin{array}{l}\text { Shima et al } \\
2019^{12}\end{array}$ & $68 \mathrm{M}$ & $\begin{array}{l}\text { Creatinine } 0.75 \mathrm{mg} / \mathrm{dL} \\
\text { eGFR } 110 \mathrm{~mL} / \mathrm{min} / 1.73 \mathrm{~m}^{2}\end{array}$ & 14 months & $\begin{array}{l}\text { Creatinine } 1.55 \mathrm{mg} / \mathrm{dL} \\
\text { eGFR } 48 \mathrm{~mL} \\
\mathrm{~min} / 1.73 \mathrm{~m}^{2}\end{array}$ & Confirmed & $\begin{array}{l}\text { Discontinued } \\
\text { alogliptin }\end{array}$ & $\begin{array}{l}\text { Partial recovery } \\
-3 \text { weeks }\end{array}$ \\
\hline \multirow[t]{2}{*}{ Sulfonylureas } & Gliclazide & $\begin{array}{l}\text { Oyama et al } \\
2018^{13}\end{array}$ & \multicolumn{7}{|c|}{$\begin{array}{l}\text { Retrospective study using spontaneous reporting system databased. Based on 5,195,890 reports of all adverse drug reactions, } \\
3,088 \text { reports of drug-induced tubulointerstitial nephritis were evaluated. Results suggested that gliclazide had the highest re- } \\
\text { porting odds ratio of tubulointerstitial nephritis }\end{array}$} \\
\hline & Glimepiride & $\begin{array}{l}\text { Akbar et al } \\
2010^{14}\end{array}$ & $50 \mathrm{M}$ & Unreported & Unreported & $\begin{array}{l}\text { Creatinine } 2.72 \mathrm{mg} / \mathrm{dL} \\
\text { eGFR } 32 \mathrm{~mL} / \mathrm{min} / 1.73 \mathrm{~m}^{2}\end{array}$ & Confirmed & $\begin{array}{l}\text { Discontinued } \\
\text { glimepiride. } \\
\text { Prednisolone }\end{array}$ & $\begin{array}{l}\text { Partial recovery } \\
\text { - few weeks }\end{array}$ \\
\hline \multirow[t]{2}{*}{$\begin{array}{l}\text { Thiazolidine- } \\
\text { diones }\end{array}$} & Rosiglitazone & $\begin{array}{l}\text { Castledine } \\
\text { et al } 2006^{15}\end{array}$ & $55 \mathrm{M}$ & $\begin{array}{l}\text { Creatinine } 97 \mu \mathrm{mol} / \mathrm{L} \\
\text { eGFR } 97 \mathrm{~mL} / \mathrm{min} / 1.73 \mathrm{~m}^{2}\end{array}$ & 3 weeks & $\begin{array}{l}\text { Creatinine } 458 \mu \mathrm{mol} / \mathrm{L} \\
\text { eGFR } 12 \mathrm{~mL} / \mathrm{min} / 1.73 \mathrm{~m}^{2}\end{array}$ & Confirmed & $\begin{array}{l}\text { Discontinued } \\
\text { rosiglitazone. } \\
\text { Prednisolone }\end{array}$ & $\begin{array}{l}\text { Partial recovery } \\
-6 \text { months }\end{array}$ \\
\hline & Rosiglitazone & $\begin{array}{l}\text { Ghani et al } \\
2009^{16}\end{array}$ & $65 \mathrm{M}$ & $\begin{array}{l}\text { Creatinine } 150 \mu \mathrm{mol} / \mathrm{L} \\
\text { eGFR } 43 \mathrm{~mL} / \mathrm{min} / 1.73 \mathrm{~m}^{2}\end{array}$ & 2 weeks & $\begin{array}{l}\text { Creatinine } 1474 \mu \mathrm{mol} / \mathrm{L} \\
\text { eGFR } 3 \mathrm{~mL} / \mathrm{min} / 1.73 \mathrm{~m}^{2}\end{array}$ & Confirmed & $\begin{array}{l}\text { Discontinued } \\
\text { rosiglitazone. } \\
\text { Haemodialysis. } \\
\text { Mycophenolate } \\
\text { mofetil. }\end{array}$ & $\begin{array}{r}\text { Partial recovery } \\
-6 \text { weeks }\end{array}$ \\
\hline
\end{tabular}


to experience a progressive decline in eGFR of $1.9-3.3 \mathrm{~mL} / \mathrm{min} / 1.73$ $\mathrm{m}^{2}$ per year. ${ }^{13}$ In contrast, our case experienced a decline in eGFR of $18 \mathrm{~mL} / \mathrm{min} / 1.73 \mathrm{~m}^{2}$ within 5 months. Due to the time correlation between eGFR decline and initiation of liraglutide, it was highly suggestive that this decline was due to liraglutide therapy; we could not determine any other reason for such a rapid decline in eGFR.

There have been similar case reports to ours in the literature where use of liraglutide (and other antidiabetic medications) resulted in acute interstitial nephritis (Table 2). These cases, like ours, experienced no gastrointestinal symptoms thus no dehydration, yet deterioration in kidney function was evident. Renal biopsy supported the diagnosis.

It is speculated whether this injury results from an immunological response. Pathogenesis of drug-induced acute interstitial nephritis is thought to occur from type IV delayed hypersensitivity reaction to the offending medication. This can happen within days or months of exposure to the medication in question. It is unclear exactly how this process occurs, however suspected mechanisms include molecular mimicry or direct binding of hapten drug to tubular membrane, resulting in an immunogenic response. ${ }^{12}$ Furthermore, antibody production has been shown to occur after liraglutide introduction ( $8.5 \%$ of cases). ${ }^{14}$

Current standard of care for treatment of drug-induced acute interstitial nephritis involves early recognition of the culprit drug and discontinuation of the medication. Late recognition of kidney damage and continued drug use may result in kidney fibrosis, with $40-60 \%$ of people with acute tubulointerstitial nephritis ultimately developing chronic kidney disease. ${ }^{8}$ Corticosteroid therapy is controversial in the treatment of drug-induced acute interstitial nephritis. Some studies report rapid and complete recovery of baseline renal function in those treated with steroids, ${ }^{15,16}$ whilst others have failed to confirm these findings. ${ }^{17,18}$ No prospective randomised controlled trials investigating corticosteroid treatment in acute interstitial nephritis have been conducted as yet. Multicentre prospective randomised controlled trials are needed to study the effect of corticosteroid therapy on interstitial nephritis. Nevertheless, the main conclusion from all studies investigating steroid treatment is that the earlier steroid treatment was initiated, the better the prognosis. ${ }^{19}$

The situation is further complicated when using steroids to treat acute interstitial nephritis in people with diabetes due to glucocorticoid-induced hyperglycaemia. ${ }^{20}$ Furthermore, it has been noted in the literature that people with diabetes are less likely to respond to steroid treatment. In addition, interstitial fibrosis in renal biopsy is associated with poor response to steroids. ${ }^{21}$ This may be due to fibrosis indicating irreversible damage of renal tissue. In our case, as significant interstitial fibrosis and tubular atrophy was noted on renal biopsy, it was deemed that steroid therapy would not be beneficial. Furthermore, with our patient's diabetes under stable control, steroid therapy was avoided to ensure $\mathrm{HbA}_{1 \mathrm{c}}$ did not deteriorate.

Of interest, liraglutide and other GLP-1 agonists have been reported to cause acute kidney injury via a different pathogenesis. Those affected severely by the gastrointestinal side effect of liraglutide treatment may experience dehydration and progress to acute

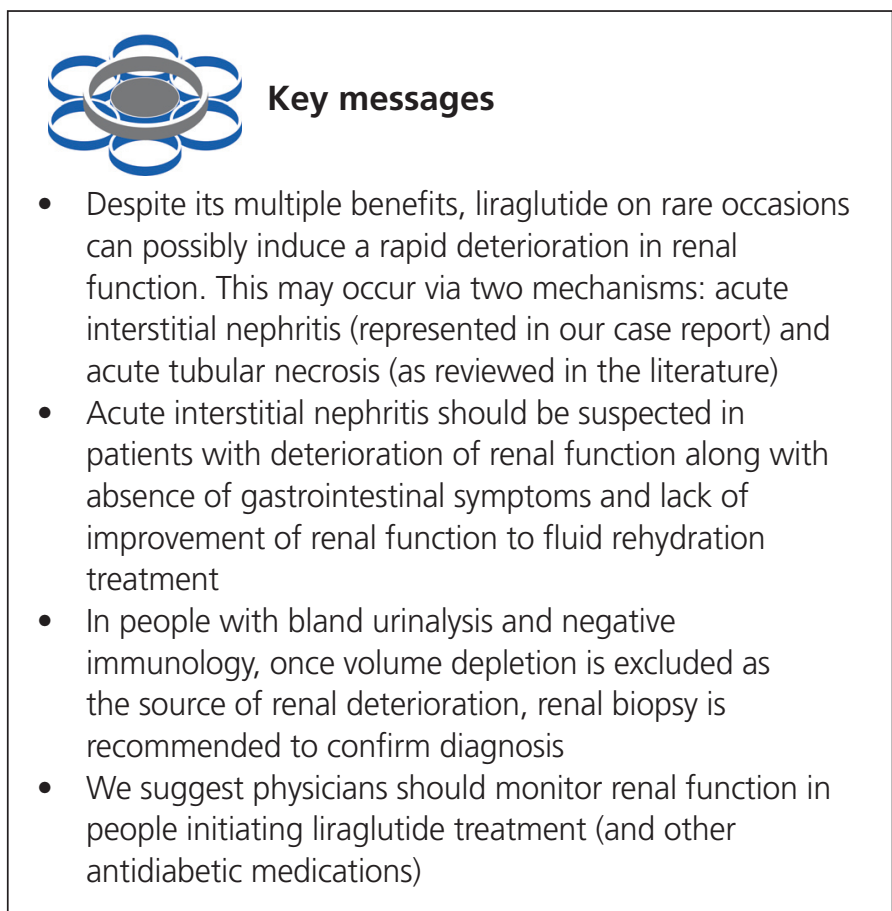

kidney injury. In these patients, renal biopsy confirms acute tubular necrosis. Careful fluid balance and examination is necessary to determine intravascular volume depletion. Fluid rehydration is essential in the treatment of these patients. ${ }^{22,23}$ This represents a diagnostic challenge for physicians, whereby clinical and laboratory features are comparable for both acute tubulointerstitial nephritis due to medication and acute tubular injury due to dehydration. ${ }^{24}$ Especially in people with diabetes, the complexity for diagnosis of renal disease is challenging. However, as our case experienced no gastrointestinal side effects, had stable weight and blood pressure and was clinically euvolaemic throughout liraglutide treatment, it was unlikely she had kidney function deterioration via this mechanism. Histological investigation further confirmed our suspicions, and excluded volume depletion as a cause of her eGFR deterioration.

Despite our case report, we would like to highlight the multiple studies reporting the benefit of liraglutide on metabolic, cardiovascular and renal outcomes. ${ }^{2-4}$ Furthermore, post hoc analysis of people with CKD have further shown the safety and efficacy of liraglutide treatment, and its benefits in reducing all-cause mortality in this patient subtype. ${ }^{25,26}$ We therefore conclude that liraglutide has a positive impact on renal function. However, physicians should be aware of acute interstitial nephritis as a possible rare side effect.

\section{Conclusion}

Despite the multiple cardiovascular and renal benefits of liraglutide therapy, our case highlights a rare side effect - acute interstitial nephritis. Few cases have been reported in the literature, thus high clinical suspicion needs to be maintained in those with rapid renal deterioration after liraglutide (and other antidiabetic medication) initiation. If interstitial nephritis is suspected and volume depletion has been excluded as a differential diagnosis, the gold standard in- 
vestigation is renal biopsy. Definitive management of antidiabetic medication-induced acute interstitial nephritis involves identification and removal of the offending medication. Steroid therapy is controversial, with a limited effect noted in those with diabetes. From our case we thus aim to raise awareness to clinicians about a rare possible side effect of liraglutide (and other antidiabetic medication) therapy and highlight its investigation and management.

Conflict of interest WA: Payment or honoraria for lectures: AstraZeneca. KG: None. NM: Payment or honoraria for lectures: Napp Pharmaceuticals, AstraZeneca, Eli Lilly; support for attending meetings: Novo, AstraZeneca. NC: Support for attending meeting: SFE BES registration grant to attend SFE BES Conference 2021. ALL: Support for attending meetings: AstraZeneca. RR: Honorarium from Eli Lilly for presentation on empagliflozin in Cardiology Department at UHCW; support for attending meetings: Napp.

\section{Funding None.}

\section{References for Table 2}

1. Gariani K, de Seigneux S, Moll S. Acute interstitial nephritis after treatment with liraglutide. Am J Kidney Dis 2014;63(2):347. https://doi.org/10.1053/ j.ajkd.2013.10.057

2. Dubois-Laforgue D, Boutboul D, Lévy DJ, et al. Severe acute renal failure in patients treated with glucagon-like peptide-1 receptor agonists. Diabetes Res Clin Pract 2014; 103(3):e53-5. https://doi.org/10.1016/j.diabres. 2013.11.014

3. Bhatti R, Flynn MD, Illahi M, et al. Exenatide associated renal failure. Pract Diabetes Int 2010;27(6):232-4.

4. Nandakoban $\mathrm{H}$, Furlong TJ, Flack JR. Acute tubulointerstitial nephritis following treatment with exenatide. Diabet Med 2013;30(1):123-5. https://doi.org/10.1111/j.14645491.2012.03738.x

5. Leehey DJ, Rahman MA, Borys E, et al. Acute kidney injury associated with semaglutide. Kidney Med 2021:3(2):282-5. https://doi.org/10.1016/ j.xkme.2020.10.008

6. Taylor SR, Moody MT. Acute kidney injury occurring in a patient initiated on dulaglutide. J Pharm Technol 2018;34(5):231-2. https://doi.org/ 10.1177/8755122518782155

7. Ryan R, Choo S, Willows J, et al. Acute interstitial nephritis due to sodium-glucose cotransporter 2 inhibitor empagliflozin. Clin Kidney J 2020;14(3): 1020-2. https://doi.org/ 10.1093/ckj/sfaa033

8. Bnaya A, Itzkowitz E, Atrash J, et al. Acute intersitital nephritis related to empagliflozin. Nephrol Dialysis Transplant 2020;35(Suppl 3). https://doi.org/10.1093/ndt/gfaa142. P0580

9. Gribben WB, Decker G. Canagliflozin-induced acute interstitial nephritis. J Hosp Med 2021:11(Suppl 1).

10. Lin DY, Hoffman D, Pulivarti W, Abendroth CS, Pulivarti B. Sitagliptin caused acute drug induced interstitial nephritis. Consultant 2014;54(6): 444-6.

11. Alsaad AA, Dhannoon SM, Pantin SA, et al. Rare allergic reaction of the kidney: sitagliptin-induced acute tubulointerstitial nephritis. BMJ Case Rep 2016;2016: bcr2016216297. https://doi.org/10.1136/bcr-2016-216297

12. Shima $H$, Okamoto $T$, Tashiro $M$, et al. Alogliptin-induced minimal change nephrotic syndrome and interstitial nephritis. Kidney Med 2019;1(2):75-8. https://doi.org/ 10.1016/j.xkme.2019.03.001

13. Oyama S, Hosohata K, Inada A, et al. Drug-induced tubulointerstitial nephritis in a retrospective study using spontaneous reporting system database. Ther Clin Risk Manag 2018:14:1599-604. https://doi.org/ 10.2147/TCRM.S168696

14. Akbar S, Goldblat M, Palekar S, et al. A rare case of interstitial nephritis with secondary membranous glomerulopathy due to glimepiride. Am J Kidney Dis 2010;55(4);836. https://doi.org/10.1053/j.ajkd.2010.02.027

15. Castledine $C$, Wright $D$, Kingdon E. Rosiglitazone as a cause of acute interstitial nephritis. Nephrol Dial Transplant 2006;21(7):1994-5. https://doi.org/10.1093/ ndt/gfl045

16. Abdul Ghani R, Zainudin S, Kamaruddin NA, et al. Acute renal failure following the use of rosiglitazone in a chronic kidney disease patient. Singapore Med J 2009;50(1):e324.

\section{References}

1. MacDonald PE, El-Kholy W, Riedel MJ, Salapatek AM, Light PE, Wheeler MB. The multiple actions of GLP-1 on the process of glucose-stimulated insulin secretion. Diabetes 2002;51(Suppl 3):S434-42. https://doi.org/ 10.2337/ diabetes.51.2007.s434

2. Yin WL, Bain SC, Min T. The effect of glucagon-like peptide-1 receptor agonists on renal outcomes in type 2 diabetes. Diabetes Ther 2020; 11(4):835-44. https://doi.org/10.1007/s13300-020-00798-x

3. Marso SP, Daniels GH, Brown-Frandsen K, et al; LEADER Steering Committee; LEADER Trial Investigators. Liraglutide and cardiovascular outcomes in type 2 diabetes. N Engl J Med 2016;375(4):311-22. https://doi.org/ 10.1056/NEJMoa1603827

4. Mann JFE, Ørsted DD, Brown-Frandsen K, et al; LEADER Steering Committee and Investigators. Liraglutide and renal outcomes in type 2 diabetes. $N$ Engl J
Med 2017:377(9):839-48. https://doi.org/10.1056/NEJMoa1616011

5. Davies MJ, Bain SC, Atkin SL, et al. Efficacy and safety of liraglutide versus placebo as add-on to glucose-lowering therapy in patients with type 2 diabetes and moderate renal impairment (LIRA-RENAL): a randomized clinical trial. Diabetes Care 2016;39(2):222-30. https://doi.org/10.2337/dc14-2883

6. Gariani K, de Seigneux S, Moll S. Acute interstitial nephritis after treatment with liraglutide. Am J Kidney Dis 2014;63(2):347. https://doi.org/10.1053/j.ajkd. 2013.10.057

7. Goicoechea M, Rivera F, López-Gómez JM; Spanish Registry of Glomerulonephritis. Increased prevalence of acute tubulointerstitial nephritis. Nephrol Dial Transplant 2013;28(1):112-5. https://doi.org/10.1093/ndt/gfs143

8. Moledina DG, Perazella MA. Treatment of drug-induced acute tubulointerstitial nephritis: the search for better evidence. Clin J Am Soc Nephrol 2018;13(12): 1785-7. https://doi.org/10.2215/CJN.12001018

9. Moledina DG, Perazella MA. Drug-induced acute interstitial nephritis. Clin J Am Soc Nephro/ 2017;12(12):2046-9. https://doi.org/10.2215/ CJN.07630717

10. Shah S, Carter-Monroe N, Atta MG. Granulomatous interstitial nephritis. Clin Kidney J 2015;8(5):516-23. https://doi.org/10.1093/ckj/sfv053

11. Perazella MA. Clinical approach to diagnosing acute and chronic tubulointerstitial disease. Adv Chronic Kidney Dis 2017;24(2):57-63. https://doi.org/ 10.1053/j.ackd.2016.08.003

12. Perazella MA, Markowitz GS. Drug-induced acute interstitial nephritis. Nat Rev Nephrol 2010;6(8):461-70. https://doi.org/10.1038/nrneph.2010.71

13. Vistisen D, Andersen GS, Hulman A, et al. Progressive decline in estimated glomerular filtration rate in patients with diabetes after moderate loss in kidney function - even without albuminuria. Diabetes Care 2019;42(10):1886-94. https://doi.org/10.2337/dc19-0349

14. Buse JB, Garber A, Rosenstock J, et al. Liraglutide treatment is associated with a low frequency and magnitude of antibody formation with no apparent impact on glycemic response or increased frequency of adverse events: results from the Liraglutide Effect and Action in Diabetes (LEAD) trials. J Clin Endocrinol Metab 2011;96(6):1695-702. https://doi.org/ 10.1210/jc.2010-2822

15. Ramachandran R, Kumar K, Nada R, et al. Drug-induced acute interstitial nephritis: a clinicopathological study and comparative trial of steroid regimens. Indian J Nephrol 2015;25(5):281-6. https://doi.org/10.4103/0971-4065. 147766

16. Prendecki M, Tanna A, Salama AD, et al. Long-term outcome in biopsy-proven acute interstitial nephritis treated with steroids. Clin Kidney J 2017; 10(2):2339. https://doi.org/10.1093/ckj/sfw116

17. Clarkson MR, Giblin L, O'Connell FP, et al. Acute interstitial nephritis: clinical features and response to corticosteroid therapy. Nephrol Dial Transplant 2004;19(11):2778-83. https://doi.org/10.1093/ndt/gfh485

18. Valluri A, Hetherington $L$, Mcquarrie $E$, et al. Acute tubulointerstitial nephritis in Scotland. QJM 2015;108(7):527-32. https://doi.org/10.1093/ qjmed/hcu236

19. Muriithi AK, Leung N, Valeri AM, et al. Biopsy-proven acute interstitial nephritis, 1993-2011: a case series. Am J Kidney Dis 2014;64(4):558-66. https://doi.org/ 10.1053/j.ajkd.2014.04.027

20. Tamez-Pérez HE, Quintanilla-Flores DL, Rodríguez-Gutiérrez R, et al. Steroid hyperglycemia: prevalence, early detection and therapeutic recommendations: a narrative review. World J Diabetes 2015;6(8):1073-81. https://doi.org/10.4239/ wjd.v6.i8.1073

21. Surendra M, Raju S, Chandragiri S, et al. Steroid therapy in drug induced acute interstitial nephritis: retrospective analysis of 83 cases. Saudi J Kidney Dis Transpl 2019:30(1):157-165.

22. Dubois-Laforgue D, Boutboul D, et al. Severe acute renal failure in patients treated with glucagon-like peptide-1 receptor agonists. Diabetes Res Clin Pract 2014;103(3):e53-5. https://doi.org/10.1016/j.diabres.2013.11.014

23. Bhatti R, Flynn MD, Illahi M, et al. Exenatide associated renal failure. Pract Diabetes Int 2010;27(6):232-4.

24. Moledina DG, Parikh CR. Differentiating acute interstitial nephritis from acute tubular injury: a challenge for clinicians. Nephron 2019;143(3):211-16. https://doi.org/10.1159/000501207

25. Mann JFE, Fonseca $V$, Mosenzon O, et al. Effects of liraglutide versus placebo on cardiovascular events in patients with type 2 diabetes mellitus and chronic kidney disease. Circulation 2018;138(25):2908-18. https://doi.org/10.1161/ CIRCULATIONAHA.118.036418

26. Mann JFE, Fonseca VA, Poulter NR, et al; LEADER Trial Investigators. Safety of liraglutide in type 2 diabetes and chronic kidney disease. Clin J Am Soc Nephro/ 2020;15(4):465-73. https://doi.org/10.2215/CJN.11881019 